\title{
Process-Based Information Systems Development: Taking Advantage of a Component-Based Infrastructure
}

\author{
José Luís Pereira, Jorge Oliveira e Sá
}

Information Systems Department and Algoritmi Research Centre, University of Minho, Portugal

\section{Abstract}

Background: Owing to the highly competitive business environment in which contemporary organizations have to operate, a quick and effective way of developing and maintaining information systems is of utmost importance to their success. Obviously, the computerized information systems that support the everyday operations and management of organizations play a critical role in their competitiveness. For that reason, nowadays, more than ever, information systems have to be quickly developed, rapidly reconfigured, and easily maintained. Objectives: We aim to define a technological infrastructure, accompanied by a set of methodological development requirements, which might help to fulfil those needs. Methods/Approach: In this work, we followed a Design Science Research (DSR) approach. Results: We propose a specific IT infrastructure, inspired by the concept of a business process and using the functionalities provided by collaborative and workflow technologies, which allows the development of distributed IT solutions, Process-Based Information Systems (PBIS), in a componentbased fashion. In the context of PBIS, we also propose a set of development requirements. Conclusions: We claim that Process-Based Information Systems allow organizations to evolve quickly and smoothly in face of changing business requirements, facilitating the integration of existing and future IT artefacts, while simplifying the overall development and maintenance effort of information systems.

Keywords: business process; BPMS; workflow technology; collaborative applications JEL classification: $\mathrm{O} 33$

Paper type: Research article

Received: March 12, 2017

Accepted: Jun 24, 2017

Citation: Pereira, J.L., Sá, J.O. (2017), "Process-Based Information Systems Development: Taking Advantage of a Component-Based Infrastructure", Business Systems Research, Vol. 8, No. 2, pp. 71-83.

DOI: 10.1515/bsrj-2017-0017

\section{Introduction}

The turbulent and increasingly competitive markets in which modern organizations have to operate and do business requires from their computerized information systems the ability of being quickly developed, rapidly reconfigured, and easily 
maintained (Pereira, 2014). These qualities are of extreme importance as IT solutions play a critical role in the competitiveness of organizations. In fact, everyone agrees that, in the global and knowledge intensive society we live in, organizations compete, more and more, in terms of their agility in reacting timely and adequately to the market pressures and opportunities (Rosemann and vom Brocke, 2015). Obviously, the computerized information systems that support the everyday operations and management of organizations play a critical role in this environment.

In addition to these demanding market conditions, organizations also have to deal with an increasingly complex IT environment, where new technologies are continually appearing on the landscape. Of course, it is necessary that organizations make a creative use of the new technological possibilities in their information systems development efforts, so that they can remain competitive.

Still in the technological domain, due to the widespread use of personal computers and the so-called commodity hardware, associated to the huge advances in the data communications field, the monolithic data processing solutions of the past, based on expensive computer platforms, are being quickly replaced by sets of powerful and cheap computers (Pereira, 2014). These, associated with a myriad of computer peripherals, interconnected by data networks, originate highly distributed solutions (Liao and Tu, 2007).

For that reason, technological complexity, distribution and rapidly changing requirements are the fundamental keywords in the Information Systems Development (ISD) arena of the present days. Therefore, additionally to using suitable ISD methodologies, organizations have to create the technological infrastructure that will allow them to develop and maintain, timely and effectively, their information systems, making use of all the technological possibilities that arise (Pereira and Sá, 2016).

In this paper we describe a specific IT infrastructure, inspired in the concept of business process, and largely built on the functionalities provided by collaborative/workflow technologies, which will facilitate the development of distributed IT solutions in a component-based fashion. Furthermore, we argue that this specific kind of IT solution allows organizations to evolve quickly in face of changing business requirements, and facilitates the integration of existing and new IT artefacts, simplifying the overall development and maintenance effort of information systems. In the following, we identify the major limitations of conventional ISD methodologies regarding this particular kind of systems and, therefore, we describe a set of development requirements associated to our specific proposal. Finally, we make some comments and conclude.

\section{Background}

In the next subsections the three main technologies are briefly described, which are underlying our IT infrastructure proposal.

\section{Collaborative technologies}

Because of the remarkable developments in the hardware and data communications fields, a set of technologies, globally known as collaborative technologies, has emerged. These technologies are primarily intended to support the communication and collaboration needs of people working together (Li et al., 2015). Electronic mail, videoconference, team rooms, group editors, discussion groups and workflow systems are just some examples of collaborative technologies. 
Different collaborative technologies have distinct characteristics regarding the time and space dimensions. A commonly used classification for the various collaborative technologies is Johansen's Time/Space Matrix (Johansen, 1991). This classification tries to distinguish among the different collaborative technologies, classifying them in a synchronous/asynchronous (time) and centralized/distributed (space) framework (see Figure 1).

Figure 1

Collaborative Technologies and the Johansen's Time/Space Matrix

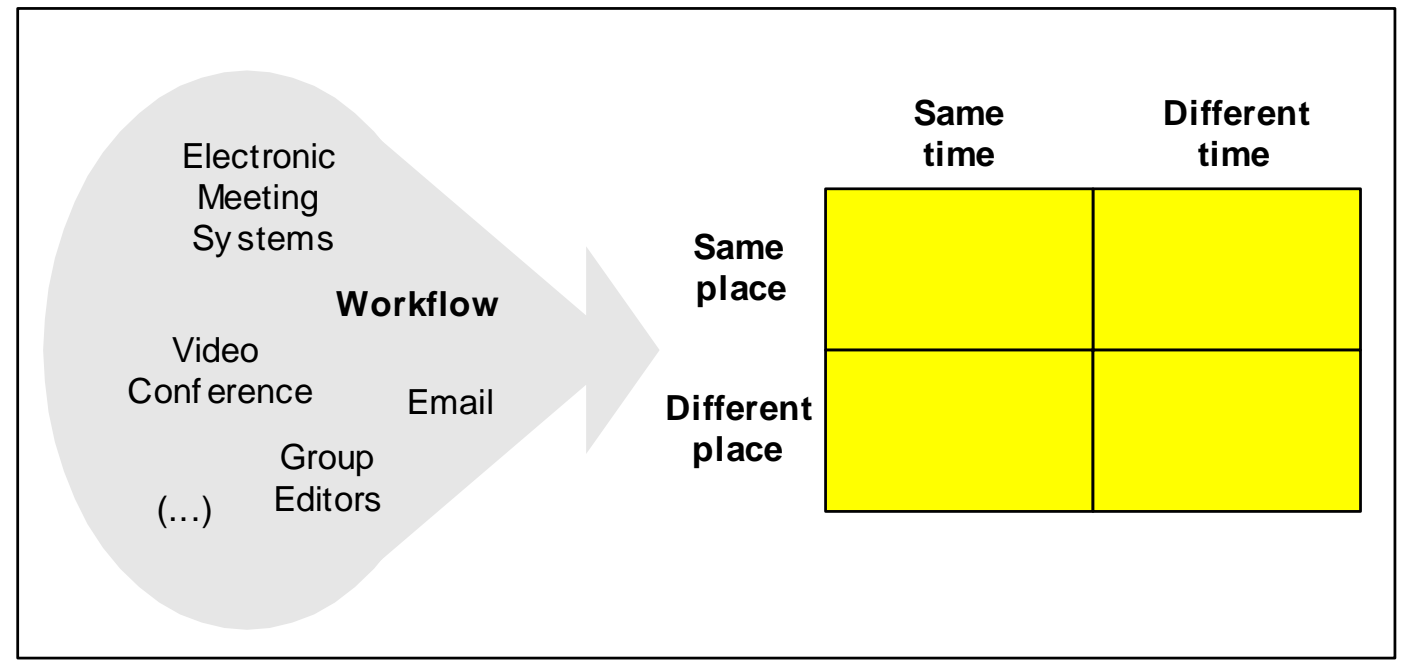

Source: Author's illustration

\section{Workflow technology}

Although workflow systems are normally considered collaborative systems, there is a subtle but very important difference between workflow and the other types of collaborative technologies. While all of them intend to facilitate the communication and collaboration among a group of people, the workflow systems aim, more specifically, to coordinate their interactions according to a particular business/organizational process (Aalst and Van Hee, 2004).

The Workflow Management Coalition (WfMC), a non-profit international body created for the development and promotion of workflow standards, defines workflow as "The automation of a business process, in whole or part, during which documents, information or tasks are passed from one participant to another for action, according to a set of procedural rules" (Workflow Management Coalition, 1999). Therefore, the notion of business process is central to workflow technology.

A widely accepted classification of workflow products distinguishes among four categories: ad hoc, collaborative, administrative and production workflow (Hollingsworth, 2004). The differences among them consist, very broadly speaking, in the more or less rigidity of the process enactment. Thus, in one extreme, we find production workflow, which aims to support the enactment of completely predefined business processes, executing them in a very rigid and strict way. Obviously, these workflow systems are adequate to support mission-critical business processes, where nothing can fail and everything must be executed according to the predefined process models. In the other extreme, we find collaborative workflow, where the focus is not so much the process per si, but the sharing of information among the people (actors) involved in the process (Becker et al., 2002). 


\section{Business Process Management Systems}

Business Process Management Systems (BPMS) are the modern software systems that implement the workflow concept by managing the execution of business processes, according to their specification models.

During the execution of a business process, the BPMS delivers work to actors (humans or machines) according to the correspondent process model and the execution context of each particular process instance. In doing this, the workflow engine of the BPMS invokes the suitable available applications with the corresponding data involved, thus creating the adequate execution context for each process activity (Dumas et al., 2013).

The success of the business process paradigm has led to the development of many commercial BPMS. These include Oracle Business Process Management Suite, TIBCO ActiveMatrix, AuraPortal, Bizagi BPM Suite, and so on. In the open source community BPMS products like jBPM, Bonita BPM, Camunda or Intalio are also very successful in the market.

One of the main characteristics of current BPMS is the implicit assumption that each process activity is an individual activity. In other words, each activity is executed by a single actor (human or machine) during a time interval. Obviously, we can think of many situations in which two or more actors have to collaborate at the same time (being in the same or different places) in the execution of the same process activity (e.g., a meeting to make a group decision). The general idea here is to relax the former constraint and be able to see a process activity also as a group activity, supported by some kind of collaborative technology.

\section{Methodology}

Among the various research methodologies used in the Information Systems field, more recently the Design Science Research (DSR) has gained wide popularity in the organizational context, due to the tangible and significant results that usually are achieved using DSR.

According to Adomavicius et al. (2008) DSR "involves the construction and evaluation of IT artefacts, constructs, models, methods, and instantiations, by which important organizational IT problems can be addressed".

The DSR approach focuses on the utility of the results, i.e., the results of the research effort have to contribute to the solution or to the improvement of a real organizational problem or, at least, have to add knowledge to the research field. Given the nature of the problem addressed in this work, it was decided to use the Design Science Research (DSR) approach.

Thus, according to the six phases of the DSR approach (Peffers et al., 2007), first we recognised a clearly significant problem, showing its relevance to organizations and the importance of a suitable solution; then we identified a set of technologies whose functionalities may help to solve/improve the situation; after that we conceive and propose a solution (Process-Based Information System) that is described in the following section.

This paper represents one of several other initiatives we are engaged in trying to "communicate" to relevant audiences the results of our work, which is the last step of the DSR approach. 


\section{Results and Discussion}

\section{The Process-Based information System Concept}

In the past there were several attempts to develop the so-called enterprise-wide information systems. These are large systems, almost inevitably distributed, which try to create a cohesive whole from dispersed components. The first serious systems integration efforts began with the advent of Data Base Management Systems (DBMS). Since the construction of a centralized repository, containing all the organizational data, was not always possible or even feasible, different solutions were engineered, such as distributed databases, in which the data was physically dispersed by several databases, but could be seen by the application level as a unique, logically integrated, repository.

This kind of systems integration may be called integration-via-data (i.e., the systems are interconnected at the data level). Other distributed solutions involve Transaction Processing Monitors to interconnect dispersed systems in the execution of distributed transactions and distributed objects interconnected by middleware technology such as web services (Chiang, 2014). Owing to the facilities provided by the collaborative technologies and, in particular workflow technology, a new possibility of systems integration has emerged, much more promising than integration via data: the integration-via-processes.

In the heart of the integration-via-processes approach are the BPMS. In fact, a BPMS may be regarded as a very sophisticated form of middleware, which more than allowing a passive interconnection between different systems allows their active interconnection, making them cooperate explicitly in the execution of a business/organizational process. Thus, a BPMS may be seen as a coordination level, which, if placed over the conventional and collaboration systems of the organization, is able to control their cooperation (Pereira, 2014). To this global solution we call Process-Based Information System (PBIS) (Figure 2).

Figure 2

The PBIS Concept

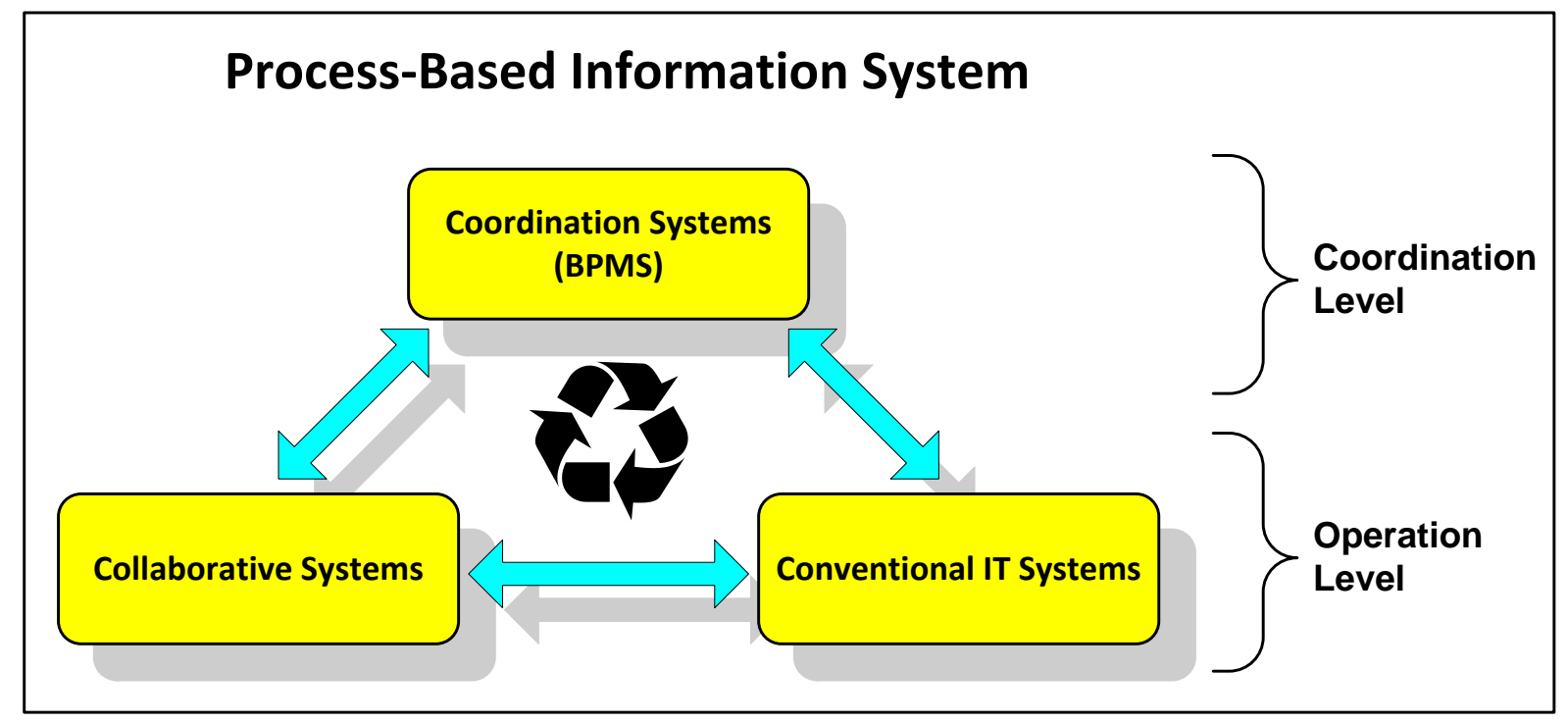

Source: Author's illustration

In the PBIS context we make a distinction between conventional systems, collaboration systems and coordination systems (Pereira and Sá, 2016). By conventional systems we mean all sorts of computer systems used to support 
individual work, such as Transaction Support Systems (TPS), Data Processing Systems (DPS), as well as Management Information Systems (MIS), Decision Support Systems (DSS), Executive Information Systems (EIS), expert systems, personal productivity tools (word processors, spreadsheets, personal databases, etc.) and, in a generic way, all the legacy information systems still in operation in organizations.

By collaboration systems we mean all sorts of computational systems used to support people working together in groups, such as videoconference systems, electronic meeting systems, electronic mail systems, etc. Conventional systems and collaboration systems are used in organizations to support people doing work in an individual or group setting, respectively. In the PBIS context, we classify these two kinds of system as operation level.

By coordination systems we mean a special kind of systems intended to control the way work flows among participants in a business process. In this group, the workflow engines of BPMS are a notorious example. In the PBIS context, these systems are used to implement the coordination level.

BPMS, besides supporting directly the process concept, have a fundamental and distinct characteristic. They allow the explicit separation between the process logic and the applications that implement the activities in the process model. Therefore, just by using this technology we are able to separate and manipulate, in an independent way, the organizational/business processes (coordination level), and the applications used to do the work (operation level).

Concerning the PBIS operation level, we distinguish among three types of work activities (Pereira and Sá, 2016)(see Figure 3):

- Automated activities, executed entirely by a machine actor, without human intervention;

- Individual activities, handled by a human actor, eventually supported by some kind of IT artefact;

- Collaborative activities, which require the collaboration of at least two human actors, in a synchronous or asynchronous way, being in the same space or geographically distributed.

Figure 3

The Three Types of Work Activities in a PBIS

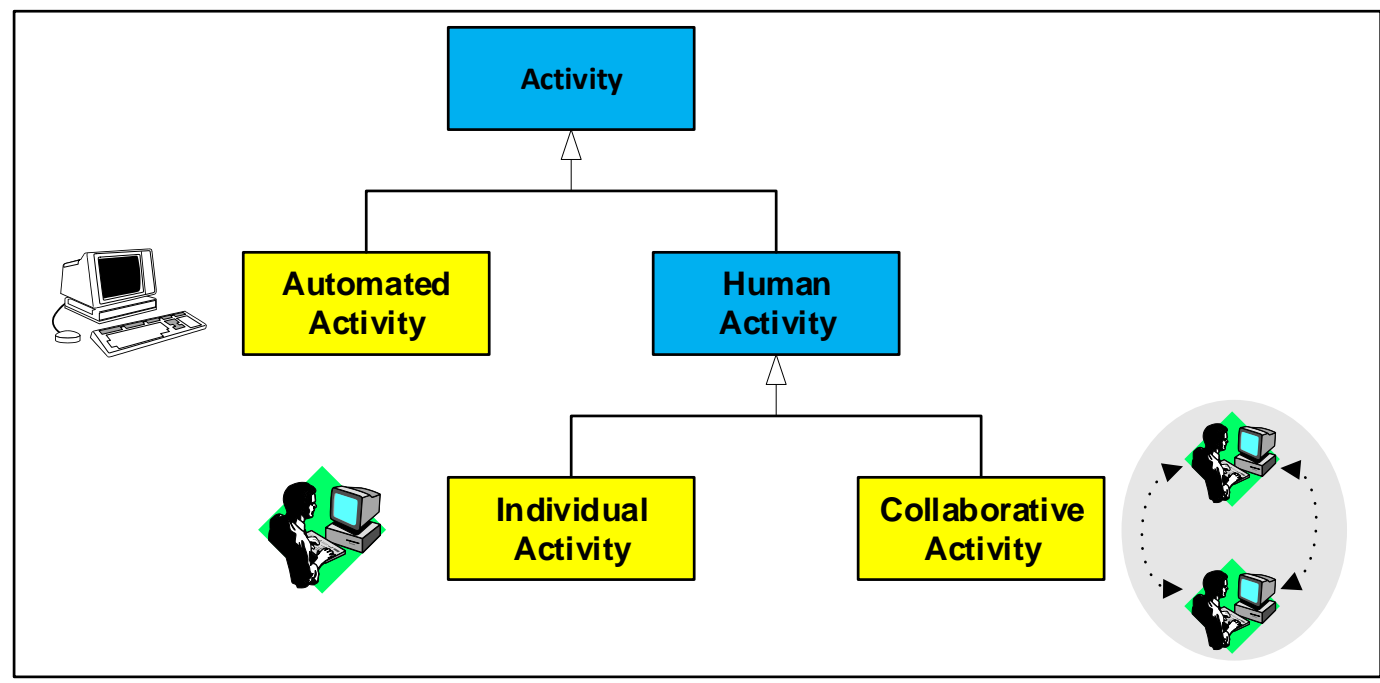

Source: Author's illustration 
The first two types of activities are good candidates to be supported by "conventional" IT. The last one (group activities) implies the use of suitable collaborative IT tools, adequate to the needs of the specific situation.

By integrating coordination systems with conventional and collaborative systems, PBIS stand as a powerful technological framework with potential to unify all the computational resources of an organization into a single global infrastructure, under the concept of business process (see Figure 4).

Figure 4

The PBIS Solution

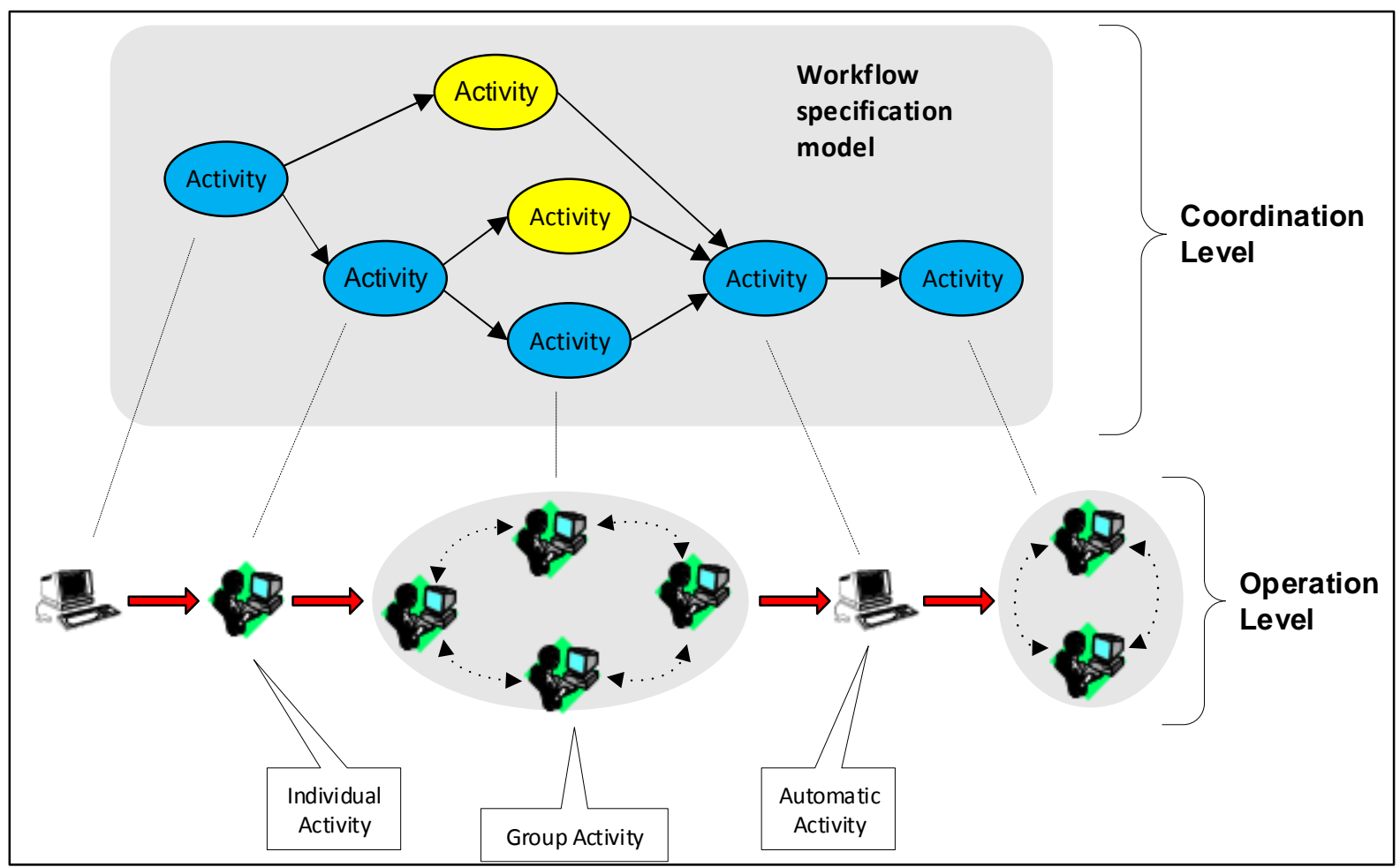

Source: Author's illustration

PBIS may be viewed as a key technological infrastructure for the development of information systems in modern organizations. It is also important to mention that the PBIS infrastructure is able to support all the dimensions of organizational work, from the more formal, routine and established work practices to the more unpredictable, social and informal ones. The PBIS infrastructure supports all these facilities in an integrated and transparent way.

The production workflow that we have mentioned earlier allows the development of typical distributed information systems (i.e., structured or formal distributed systems, where the allowed interactions among components are all predefined). With the addition of dynamic modification and collaborative facilities to BPMS, we can develop enriched kinds of distributed systems, which might be able to deal with unexpected situations, and where the informal component may be present.

\section{PBIS Development as a (Macro-) Component-Based Approach}

As we have seen before, BPMS is simultaneously a coordination technology (it allows to control the execution of other applications) and an integration technology (it behaves like a middleware technology that interconnects different systems). 
In a very simplistic way, we can identify two levels of component-based development:

- The common component-based software development, largely based on the Object-Oriented paradigm, used to develop software applications;

- The higher-level component-based systems development where complete, discrete and autonomous blocks of functionality (e.g. entire IT applications) are glued together, in a specific way, in order to build a larger system.

In the PBIS context, we call those complete IT applications macro-components. The interconnection of those macro-components is conceptually based on the notion of business process and implemented by way of BPMS (see Figure 5).

Figure 5

The Component-Based Nature of the PBIS Approach

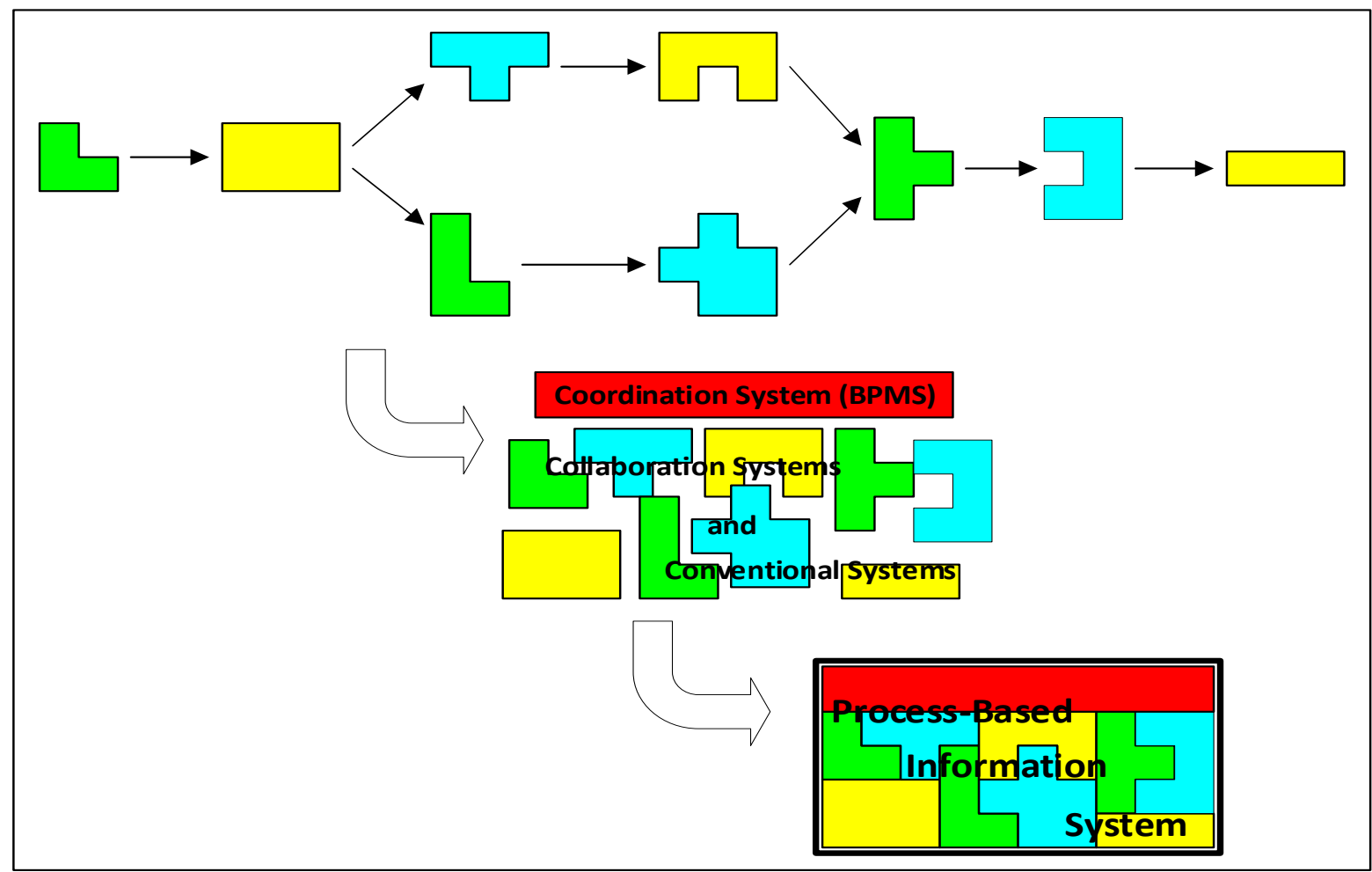

Source: Author's illustration

This approach promotes the incremental development of computer based information systems in a component-based fashion, trying to maximize the reuse of large software building blocks (IT applications). In fact, a PBIS is developed, piece by piece, in a very modular way, by adding successive process models to the coordination level, and reusing or, if not available, developing/buying new IT applications in the operation level. As time goes by, as new business processes are added to the PBIS, the level of applications' reuse will increase and, in the limit, the coordination level will be a fair representation of the corporate process model, where "all" the business processes of an organization are represented (Pereira and Sá, 2016).

Due to the component-based style of the PBIS framework, it is easier to deliver quick solutions, which are easily maintainable, thus increasing the productivity of the information systems development professionals. On the one hand, the PBIS approach promotes the concurrent development of the different components of 
the system, which certainly very much contributes to a faster development of the whole system. On the other hand, the clear separation between the coordination level and the operation level, which is a distinct characteristic of the PBIS framework, makes it possible to change or redefine the organizational processes without affecting the existing applications, and vice-versa. Therefore, the PBIS approach results in easily maintainable and highly configurable and flexible systems.

\section{PBIS Development Requirements}

The PBIS concept embodies the integration of two distinct "worlds" that are typically used apart in organizations: the conventional computer-based information systems developed in order to support and/or automate the work of individuals; and the more recent collaborative systems, still often viewed as mere "toys" by a large number of IS/IT professionals, and frequently employed by the majority of organizations simply as a lateral and secondary work support infrastructure.

Due to the low importance that IS/IT professionals typically attribute to collaborative technologies, they almost never participate in groupware development projects. This is the reason why the introduction of collaborative technologies into organizations has been characterized by a total unplanned and mostly amateurish approach (Li et al., 2015). Obviously, this kind of practice prevents organizations from taking full advantage of the rich functionalities and high potential offered by these technologies.

We claim that collaborative technologies, in the same way as the more conventional information technologies, are going to be part of the global information systems technological infrastructure and so, they should be involved in the overall Information Systems Development (ISD) effort right from the beginning (Pereira and Sá, 2016).

Obviously, the ISD methodologies should be adapted, at every moment, to the characteristics and requirements of the organisations in which it is going to be applied, and to the emerging functionalities made available by IT. In other words, ISD methodologies must be continuously aligned with the organisational and technological circumstances.

What organizations need today, to succeed in the challenging conditions of the new markets, is an integrated approach to ISD. One which makes full use of all the IT and computational resources available, both the conventional and the emerging ones, under the concept of business process.

Bearing those issues in mind, we can depict a division of a PBIS Development project in a series of smaller and mostly independent subprojects: a workflow development project, corresponding to the PBIS coordination level; and, depending on the types of activities that appear in the PBIS operation level, several collaborative application development projects and conventional application development projects. A final project corresponds to a systems integration project, which making use of the facilities made available by BPMS, integrates the different subsystems into a global solution - the PBIS (see Figure 6).

The transformation of business process models into information systems specifications, adequate to the development of computer artefacts, is a complex process that requires a strong involvement and commitment of all stakeholders - the business agents and the IS/IT experts. As practice has shown, this is an essential prerequisite for the latter acceptance of the developed systems. In the PBIS approach, excluding the systems integration, which is a rather technical phase, business agents need to be present at all phases. 
Figure 6

The PBIS Development Approach

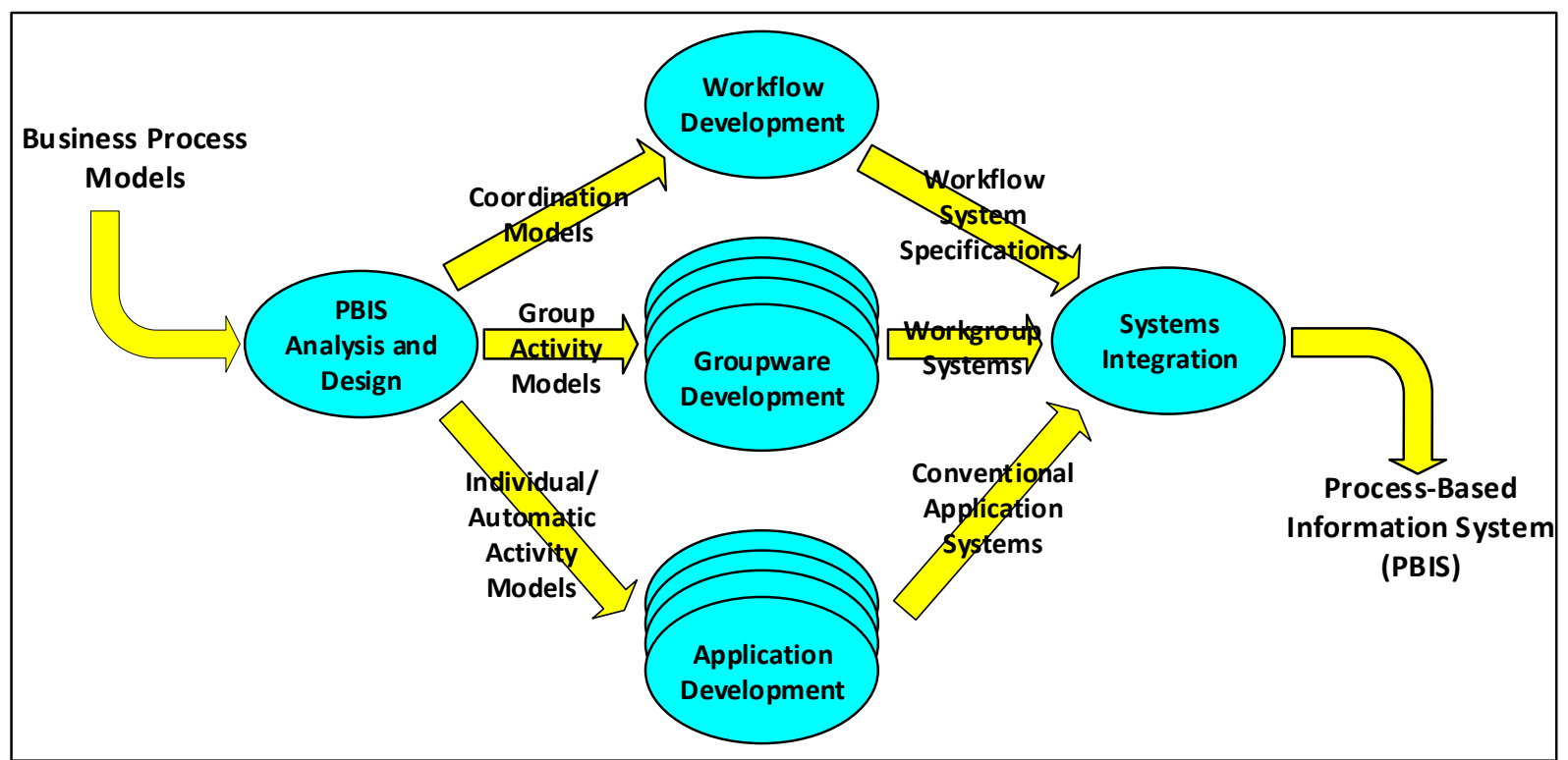

Source: Author's illustration

The first PBIS step (PBIS Analysis and Design) transforms a business process model into a set of models adequate to the development of the various PBIS components. According to Curtis et al. (1992), there are four essential modelling perspectives embodied in a process model:

- The functional perspective (what needs to be done);

- The informational perspective (which data is involved);

- The behavioural perspective (when it is done);

- The organizational perspective (who does it).

While the functional and informational perspectives are crucial to develop the conventional application systems and so, must be present in the individual and automatic activity models, the behavioural and organizational perspectives represent the coordination dimension of a PBIS and thus, will be present in the coordination models. To these four perspectives, we add three others, which represent the PBIS collaboration dimension and so, will be present in the group activity models (Pereira and Sá, 2016):

- The group perspective (who interacts with whom);

- The interaction perspective (what facilities are needed for the interaction);

- The responsibility perspective (who is responsible for the interaction).

The individual/automatic activity models represent the requirements specifications of the applications needed to automate work procedures or to support the work of individuals, respectively. Those models are used as a basis to develop the corresponding IT applications, reuse applications already existent in the organization, or select and acquire commercially available software packages (usually known as COTS - Common of the Shelf packages)

Regarding the modelling language used to express those specifications, we claim that UML (Unified Modelling Language) is a convenient requirements specification language. The principal reason is that, today, UML is viewed as the standard modelling language for software development. Another reason is that the UML set of models are methodology-neutral and so, they may be used subsequently in any 
methodology context, allowing organizations to choose freely their software development approaches (OMG, 2015).

The group activity models represent the requirements specifications of the collaborative systems intended to support group activities. It is important to note that collaborative systems are general-purpose systems, which can be employed in a multitude of situations. The contribution of PBIS Development at this level consists in the appropriate specification of the collaborative facilities needed for each work situation. These specifications will be subsequently used to select the suitable collaborative tools.

In the case of a group activity requiring the synchronous collaboration among several human actors (e.g., a meeting between two or more people to make a joint decision) it is necessary to find a moment in which the required participants (each one with his/her own agenda) can meet together, in geographically co-located or distributed fashion (Uahi and Pereira, 2016). Several criteria to select the group participants can be envisaged (e.g., the quicker group formation, manual group formation done by the participant responsible for the meeting, etc.). Although the internals of the group activity are not under the control of the coordination system, the start and end conditions are their responsibilities.

Concerning the coordination models, these are used to develop the workflow specifications. Once again, we recommend the use of a standard modelling language for specifying coordination models, independent of any specific BPMS. In this regard, the BPMN (Business Process Model and Notation), as today's de facto standard for process modelling, stands as the suitable option (OMG, 2013).

\section{Conclusion}

In this paper, we describe an innovative approach to the development of Information Systems in which the concept of business process acquires particular relevance. To the overall solution, we call Process-Based Information System (PBIS). The PBIS approach promotes the incremental development of Information Systems, in which the whole solution is developed piece by piece, in a very modular way, by adding successive workflow models to the coordination level, and reusing or, if not available, developing/acquiring new applications and groupware facilities in the operation level. In a general view, the PBIS approach might be regarded as a kind of component-based Information System Development (ISD), by analogy with the component-based software development in the software engineering domain. A kind of "programming in the large", where complete software components, corresponding to autonomous applications and groupware facilities, are combined and put to work together according to some business process specification.

It is widely recognized that organizations have always complained about their ISD projects. Usually, solutions were delivered too late, too costly and sometimes lacking important requirements, which triggered several research efforts in the past, in order to conceive new ISD methodologies. Today, more than ever, organizations need to use agile approaches to ISD. Indeed, due to the rapid pace at which things change today, to maintain Information Systems continuously aligned with the needs of the business, modern ISD methodologies have to be fast at delivering suitable solutions. The PBIS approach, because of its component-based style, may quickly deliver suitable and easily maintainable solutions. On the one hand, the PBIS promotes the concurrent development of the different components of the system, which certainly very much contributes to a faster development of the whole solution. On the other hand, the clear separation between the coordination level and the operation level, which is a distinct characteristic of the PBIS approach, makes it possible to change 
or redefine the business processes without affecting the existing applications, and vice-versa. Therefore, PBIS stand easily maintainable and highly configurable.

The PBIS approach also considerably facilitates the management of large ISD projects. Indeed, by dividing a large system into a set of subsystems, which may be independently developed or acquired, an otherwise complex project will result in a series of small (sub)projects, much more easily managed. In fact, the activities included in the process models are treated as independent components ("black boxes"), specified and developed or acquired independently from each other, eventually by distinct teams, and at different moments. Obviously, this will greatly simplify the management of ISD projects.

This paper proposes PBIS as a contribution to fulfil the need of more agile approaches to the development of Information Systems, which is fundamentally based in the concept of business process, making use of the functionalities available in BPMS/workflow and collaborative technologies. While our experience in the field has already showed us the potential of the PBIS approach, we need more work that is empirical in order to consolidate the proposal. That is what we intend to do in the future.

\section{References}

1. Aalst, W. V., Van Hee, K. M. (2004). Workflow Management: Models, Methods, and Systems, The MIT Press.

2. Adomavicius, G., Bockstedt, J. C., Gupta, A., Kauffman, R. J. (2008), "Making sense of technology trends in the information technology landscape: A design science approach", MIS Quarterly, Vol. 32, No. 4, pp. 779-809.

3. Becker, J., zur Muehlen, M., Gille, M. (2002), "Workflow application architectures: classification and characteristics of workflow-based information systems", in Layna Fischer (Ed.), Workflow Handbook 2002, Future Strategies, Lighthouse Point, pp. 39-50.

4. Chiang, C. C. (2014), "Software development concerns in the building of serviceoriented based enterprise systems", in Proceedings of the 15th IEEE/ACIS International Conference on Software Engineering, Artificial Intelligence, Networking and Parallel/Distributed Computing, Las Vegas, USA.

5. Curtis, B., Kellner, M., Over, J. (1992), "Process modeling", Communications of the ACM - Special issue on analysis and modeling in software development, Vol. 35 , No. 9, pp. 75-90.

6. Dumas, M., La Rosa, M., Mendling, J., Reijers, H.A. (2013). Fundamentals of Business Process Management, Berlin Heidelberg, Springer.

7. Hollingsworth, D. (2004), "The Workflow Reference Model: 10 Years On", in Fischer, L. (Ed.), Workflow Handbook 2004, Future Strategies Inc.

8. Johansen, R. (1991), "Groupware: Future Directions and Wild Cards", Journal of Organizational Computing, Vol. 1, No. 2, pp 219-227.

9. Li, L., Guo, R., Zheng, J. (2015), "Assessing and Promoting Groupware for Effective Team Collaboration - A comprehensive Study", in Proceedings of the Southern Association for Information Systems Conference, Hilton Head Island, SC, USA.

10. Liao, K., Tu, Q. (2007), "Leveraging automation and integration to improve manufacturing performance under uncertainty: An empirical study", Journal of Manufacturing Technology Management, Vol. 19, No. 1, pp. 38-51.

11. OMG (2013), "Business Process Model and Notation 2.0.2 (BPMN 2.0.2.)", available at: http://www.omg.org/spec/BPMN/2.0.2/PDF (10 April 2017).

12. OMG (2015), "Unified Modeling Language 2.5 (UML 2.5)", available at: http://www.omg.org/spec/UML/2.5/PDF (10 April 2017). 
13. Peffers, K., Tuunanen, T., Rothenberger, M., Chatterjee, S. (2007), "A Design Science Research Methodology for Information Systems Research", Journal of Management Information Systems, Vol. 24, No. 3, pp. 45-78.

14. Pereira, J. L. (2014), "Process-based Information Systems: A Component-based Systems Development Infrastructure", in Proceedings of the 3th International Conference on Virtual and Networked Organizations, Emergent Technologies and Tools, Póvoa de Varzim, Portugal.

15. Pereira, J. L., Sá, J. O. (2016), "The Development of Process-Based Information Systems: Methodological Requirements", in Proceedings of the 27th International Business Information Management Association Conference, Milan, Italy.

16. Rosemann, M., vom Brocke, J. (2015), "The six core elements of business process management", In vom Brocke, J., Rosemann, M. (eds.), Handbook on Business Process Management, Vol. 1, Berlin Heidelberg, Springer, pp. 107-122.

17. Uahi, R., Pereira, J. L. (2016), "Task Allocation in Business Process supported by BPMS: Optimization Perspectives", in Proceedings of the 11 th Iberian Conference on Information Systems and Technologies, Gran Canaria, Spain.

18. Workflow Management Coalition (1996), "Terminology \& Glossary", available at: http://www.aiai.ed.ac.uk/project/wfmc/ARCHIVE/DOCS/glossary/glossary.html (20 September 2017).

\section{About the authors}

José Luís Pereira has a PhD in Information Systems and Technology and an MSc in Information Systems Management from the University of Minho (UMinho), in Portugal. Currently, he is an Assistant Professor in the Information Systems Department of the UMinho Engineering School. As a researcher, he is a member of the ALGORITMI Research Centre, working in the Simulation, Business Process Management (BPM) and Big Data fields, with specific interests in Decision Support, Business Process Improvement and Big Data solutions. He can be contacted at jlmp@dsi.uminho.pt.

Jorge Oliveira e Sá is an Assistant Professor in the Information Systems Department of the University of Minho (UMinho), in Portugal, and a researcher at ALGORITMI Research Centre. He has a PhD in Information Systems Technologies from UMinho in 2010. His research interests include Business Intelligence, Data Warehousing, Big Data Warehousing, Business Process Management and Corporate Process Management. He can be contacted at jos@dsi.uminho.pt. 